\title{
El diagnóstico de la gestión pública y la necesidad de implementar indicadores de desempeño organizacional conmensurables
}

\section{Diagnosis of public management and the need to implement measurable organizational performance indicators}

Cleida Incacutipa Limachi*

\begin{abstract}
Resumen
El presente artículo analiza la administración pública de corte tradicional burocrático: las debilidades en cuanto a sus instrumentos de evaluación, los resultados poco favorables para los usuarios del servicio público y la carencia de instrumentos suficientes para una adecuada valoración, a la vez que explora la posibilidad de modificación de esta realidad. El análisis lleva a plantear la urgente necesidad de construir e implementar indicadores conmensurables, que permita medir los logros de la gestión, identificar las debilidades para plantear alternativas de potenciación o de solución, haciendo una gestión más cercana al usuario. La metodología utilizada fue el cualitativo exploratorio de revisión documentaria, a partir de la cual se plantean posibilidades de construcción de indicadores adecuados a una organización para una mejor gestión. La conclusión fundamental es que toda acción gubernamental debe brindar un óptimo servicio.
\end{abstract}

Palabras clave: cultura organizacional, gestión pública, indicadores de desempeño, organización.

\begin{abstract}
This article seeks to analyze the weaknesses in the traditional bureaucratic public administration, which has diffuse instruments for its evaluation. The results are not very favorable for the users of the public service, who do not have sufficient instruments for an adequate evaluation, nor the possibility of modifying this reality. The analysis leads us to propose the urgent need to build and implement measurable indicators that allow measuring management achievements, identifying weaknesses in order to propose alternatives for improvement or solution, making management closer to the user. The methodology used was the exploratory qualitative documentary review, from which we propose possibilities for the construction of indicators, adapting them to an organization for a better management. The fundamental conclusion is that all governmental action is to provide an optimal service.
\end{abstract}

Keywords: organizational culture, public management, performance indicators, organization.

\section{Introducción}

Actualmente, las organizaciones sean privadas o públicas se encuentran ante grandes retos internos y externos que se derivan de su propia labor y el contexto social, cultural, económico y político en el que se desempeñan. Es importante entonces detectar las oportunidades en concordancia con las necesidades de los usuarios, beneficiarios o consumidores. Las teorías sobre el desempeño organizacional han evolucionado mucho, Allaire \& Firsirotu (1984)parten, en cuanto a sus análisis, desde un enfoque del comportamiento, para ellos existen ocho clasificaciones: la escuela funcionalista, estructural funcionalista, ecológica, histórica, estructuralista, mutual, simbólica y cognitiva, a partir de allí surgieron muchos otros teóricos que procuraron entender el comportamiento de las organizaciones, su desempeño, el involucramiento, su consistencia, además de su adaptabilidad (Martínez, 2010). Por su parte, Ramírez (2004) señala que la realidad ha cambiado y la discusión está en relación a las corrientes de management en las organizaciones, en el que aparece el desempeño organizacional como una disciplina.

*Universidad Nacional del Altiplano Puno, Perú. 0000-0003-4111-1105, e-mail: cleida@unap.edu.pe 
Sin embargo, desde enfoques más actuales, surgen preocupaciones y propuestas de distintos teóricos internacionales en la búsqueda de la eficiencia, la efectividad y la calidad de la gestión pública en el mundo y América Latina. El comportamiento organizacional, la innovación tecnológica y organizacional, los sistemas de comunicación dinámica, la competitividad y la calidad nos hace repensar sobre el desempeño organizacional, en especial del sector público.

El presente artículo tiene como objetivo distinguir los indicadores claves que contribuyen en el nivel de desempeño organizacional del sector público. Para lo cual se han revisado los planteamientos teóricos de distintos autores y se presentan partiendo desde una revisión de los antecedentes basados en la cultura organizacional, definición conceptual de los indicadores de desempeño, el análisis de su importancia en el sector público a partir de la integración de sus dimensiones.

\section{Método}

La metodología utilizada en el presente artículo fue de carácter cualitativo exploratorio, a partir de la sistematización documental de estudios dedicados a la evaluación de desempeño en la gestión pública. Los documentos fueron seleccionados por el abordaje e incidencia de estos en los indicadores de desempeño y gestión. Luego de un acucioso análisis se plantea una propuesta para el planteamiento de indicadores acordes a cada organización.

\section{Resultados y análisis}

\section{Antecedentes de desempeño y cultura organizacional}

Bédard (2004) observa el desempeño a partir de tres funciones: producción y creación, producción y seguridad y soberanía y gobierno; estos elementos contribuyen al desempeño organizacional. De otro lado, Martínez (2010) habla del management de desempeño como una posibilidad de potenciar la mejora al interior de las organizaciones y fuera de ellas. Por su parte, Ernult destaca el trabajo de las organizaciones en base a los objetivos y la relación de valor-costo, y mantiene una posición enfática de cumplir con los objetivos trazados. Asimismo, Savall y Zardet abordan el tema desde el concepto sustentable y soportable, es decir, cómo lograr una mayor responsabilidad social compatible con su capacidad como organización, siendo esta una posición bastante cercana al sector empresarial (Martínez, 2004). Vargas y Magaña consideran que es importante contar con una forma de medición, y desarrollan parámetros para medir el desempeño de funciones en el sector público, y plantean que estas mediciones no solo deben dar cuenta de los resultados de acción, sino de los efectos que esto genera, además, los resultados de este desempeño deben permitir la generación de políticas innovadoras, posición que se orienta más a la gestión pública (Ramírez, 2004).

Desde un enfoque de calidad total, Carlos de Mucha plantea que la verdadera reconversión debe iniciar desde el cambio de actitudes y comportamientos de un sistema organizacional, lo que implica repensar nuestras acciones desde dentro en sintonía con la realidad del entorno. A esto Deal \& Kennedy llama teoría de la cultura organizacional, basado en los fundamentos antropológicos. Así, se plantea que "la cultura de cualquier organización puede ser gerenciada hacia el logro de una mayor efectividad" (Gomez \& Rodriguez, 2015). Este análisis comprende varios aspectos que una organización debe tener en cuenta, como las características propias que la definen y distinguen de otras organizaciones similares, el mismo que implica contar con un sistema de normas de comportamiento estables en el tiempo. La ventaja que otorga la cultura a la organización es enfrentar ciertas situaciones de su medio ambiente con cierto éxito adaptativo, además, permite que a nivel organizacional las actividades están organizadas y divididas de acuerdo a sus funciones y responsabilidades y bajo un parámetro normativo y valores compartidos. En el modelo Denison de cultura organizacional se resalta cuatro características principales: el involucramiento (que destaca el empoderamiento de la gente, el desarrollo de capacidades humanas en todo los ámbitos de la organización), la consistencia (relacionada al conjunto de valores, habilidades de lograr acuerdos y las capacidades para lograr que las actividades estén coordinadas e integradas), la adaptabilidad (capacidad de responder a los cambios, de asumir riesgos, aprender de 
los errores y de crear cambios) y misión (tener el sentido claro del propósito y los objetivos estratégicos), todas ellas relacionadas a los valores, creencias y los supuestos que son difíciles de generalizar entre las organizaciones (Fondas \& Denison, 1991). Sin embargo, a través de varios estudios de experimentación con diversas empresas demostraron que la fusión cultura-desempeño dio lugar a la satisfacción de los empleados en el incremento de ventas y en los procesos de innovación, así como a la satisfacción de los clientes.

Nunca antes había sido tan importante hablar de la cultura y el desempeño organizacional. Una mirada rápida a los cambios profundos de hoy nos lleva a referir a organizaciones calificadas como modernas o hipermodernas en las que los sistemas de funcionamiento son hiperflexibles, y sus equipos poseen una brillante capacidad de diseñar, planificar y ejecutar, para esto utilizan estrategias adecuadas y precisas. Estas organizaciones, asimismo, se caracterizan por su velocidad, por su predisposición al cambio, sus competencias y habilidades, y su vigilancia a nuevas tendencias. A su vez, las mismas, crean unidades de negocio con una operatividad independiente, entornos de trabajo promotores de bienestar positivo, preparadas para enfrentar cualquier tipo de contingencias, muy disciplinadas y adiestradas en el manejo de las metodologías de sistematización que posibilita responder a su visión y misión, con programas estratégicos y tácticos que les permite cumplir sus objetivos y metas. Esta forma de organización y preparación rápidamente llevó a un crecimiento sostenido (Carr \& Hancock, 2006) a las organizaciones de países asiáticos y europeos, en contraposición a los países latinoamericanos cuyo sistema administrativo burocrático, sobre todo en el sector público, es cada vez más lento y responde solo a los intereses particulares, haciendo que nuestros sistemas de planificación, dirección, control y ejecución decaigan paulatinamente.

Frente a esta situación, tenemos a otros teóricos que han generado diversos escritos con mayores precisiones sobre todo en el ámbito de la acción pública. A partir de las recomendaciones de las políticas económicas del Consenso de Washington (1989) y las crisis macroeconómicas ocurridas se plantea una reforma administrativa del aparato estatal: transitar de una gestión burocrático-tradicional hacia un modelo de gestión pública por resultados. Este es el punto de partida de la modernización que permite medir los resultados a partir de los indicadores de desempeño. Según Bonnefoy y Armijo (2005) "la utilidad de contar con los indicadores de desempeño para los gobiernos es indiscutible"; sin embargo, el proceso de incorporación ha tomado una serie de desafíos desde su fase de diseño, planificación, control y evaluación, entre los principales se destaca la cultura organizacional, debilidades de los sistemas de servicio y el débil proceso de consolidación de los modelos burocráticos, que no permiten el funcionamiento adecuado.

\section{Definición de los indicadores de desempeño}

¿Cómo se definen los indicadores de desempeño? Para Bonnefoy y Armijo (2005) los indicadores de desempeño del gobierno están ligados a "la productividad, calidad, oportunidad, responsabilidad de los servicios y programas públicos..., implica la consideración de un proceso organizacional, dinámico en el tiempo y refleja modificaciones del entorno organizativo, de las estructuras de poder y los objetivos”, además, comprende dos conceptos fundamentales como la eficiencia y la eficacia; el primero, ligado a la habilidad de desarrollar una actividad al mínimo costo posible y, el segundo, al cumplimiento de los objetivos predefinidos, asimismo, se incluyeron criterios de desempeño presupuestario, focalización y de calidad en la prestación de los servicios públicos.

\section{Importancia de los indicadores de desempeño en el sector público}

Habitualmente, la gente tiene sueños, propósitos que buscan alcanzar, para lograrlo, se esfuerzan, trabajan duro, emplean incluso tiempo extra para tal fin; en el lado contrario, existen quienes, a pesar de tener pretensiones ambiciosas, trabajan menos, dedican menos tiempo al logro de sus propósitos y su futuro queda incierto. Situación similar sucedía en las organizaciones, aunque se plantean objetivos determinados y ambiciosos escenarios a futuro, no toman en cuenta la capacidad operativa, los 
recursos necesarios, la metodología clara, ni estrategias, ni tácticas definidas, ni las formas de medir los resultados. En la mayoría de las instituciones públicas aún no se tiene en claro la importancia y magnitud del concepto de planificación, y se comete el error de seguir "planeando" sin la certeza necesaria, y, además, se improvisan actividades fuera de alcance con la finalidad de justificar el gasto público.

La medición del desempeño no está aislada del proceso de planificación estratégica, es importante recordar que los objetivos sean de corto, mediano o largo plazo, contemplados en el plan deben ser medidos, para lo cual, con anterioridad, deberán definirse los objetivos: objetivos estratégicos, indicadores y metas alcanzables en un determinado tiempo, así como las estrategias operativas, administrativas y técnicas que permita lograr los resultados previstos. Por lo tanto:

"Los indicadores de desempeño son instrumentos de medición de las principales variables asociadas al cumplimiento de los objetivos, que a su vez constituyen una expresión cualitativa o cuantitativa concreta de lo que se pretende alcanzar con un objetivo específico establecido. (...) La evaluación del desempeño se asocia al juicio que se realiza una vez culminada la acción o la intervención. Busca responder interrogantes claves sobre cómo se ha realizado la intervención, si se han cumplido los objetivos (concretamente, la medida en que éstos han sido cumplidos), el nivel de satisfacción de la población objetivo, entre otras. En suma, se busca evaluar cuán bien o cuán aceptable ha sido el desempeño de determinado organismo público con el objetivo de tomar las acciones necesarias para perfeccionar la gestión" (Ministerio de
Economía y Finanzas del Perú, 2000). Citado por Bonnefoy y Armijo (2005).

La medición de desempeño no es una mera evaluación de los resultados de los proyectos o programas, sino forma parte, en una dimensión mayor, del rol institucional como agente de cambio, y está íntimamente relacionada a dicho rol, es decir, a su capacidad de planificar $\mathrm{y}$ ejecutar utilizando estrategias adecuadas, administrando responsablemente los recursos, cumpliendo en el tiempo previsto, vigilando los cambios y utilizando sus planes de contingencia adecuadamente y en el momento oportuno. Hay preguntas claves para esta medición: en qué medida se cumplieron los objetivos, cuál es el nivel de satisfacción de la calidad percibida por los usuarios, cuán oportunamente llegó el servicio. Lo que se busca es cuán aceptable es el desempeño de la organización.

\section{Dimensiones de los indicadores de desempeño}

Existen diversos indicadores de desempeño que las organizaciones pueden asumir para controlar o mejorar su desempeño y tomar sus decisiones. Según Gopalakrishnan el desempeño organizacional puede definirse a partir de tres aspectos: 1) la eficiencia, relacionada a los movimientos de los recursos; 2) la efectividad, relacionada con el crecimiento organizacional y la satisfacción del empleado, y, finalmente, 3) los resultados financieros, relacionados con el retorno de activos, la inversión y el crecimiento de la utilidad (Yamakawa \& Ostos, 2011). Por su parte, Bonnefoy y Armijo (2005) presenta un esquema que visibiliza un conjunto de interrelaciones entre las categorías de indicadores desde la perspectiva del proceso productivo: 


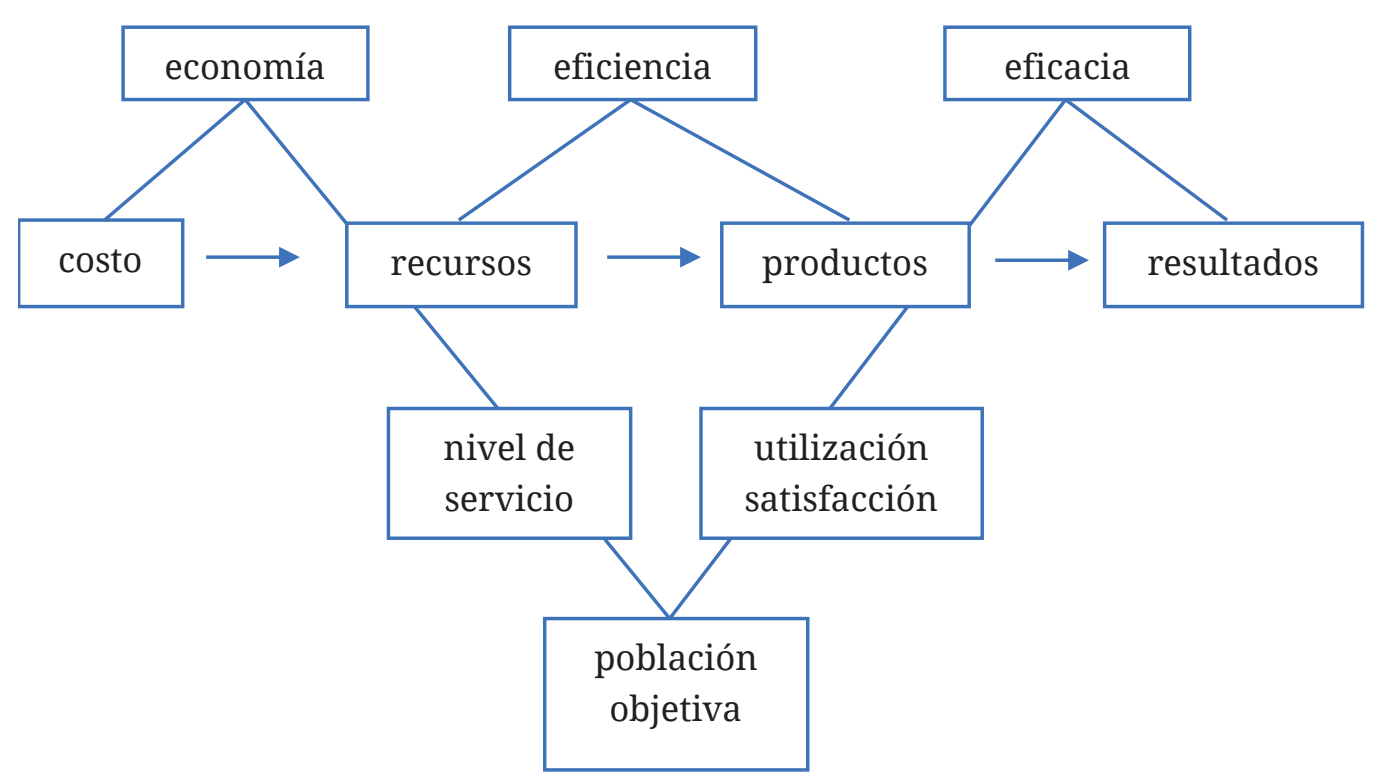

Figura 1. Interrelaciones desde la perspectiva del proceso productivo, entre economía, eficacia y eficiencia.

Fuente: Adaptado de Figura 2.2 Indicadores de Gestión para las Entidades Públicas. Asociación Española de Contabilidad y Administración de Empresas. AECA, 2002. Citada por Bonnefoy y Armijo (2005).

No hay una única forma de medir el desempeño organizacional, podemos enfocarlo desde el nivel de servicio que se ofrece a la población objetiva o desde las dimensiones de economía: eficiencia o eficacia para lograr un nivel de satisfacción. Lo más importante es lograr los objetivos que se esperan. La medición del desempeño organizacional es una constante.

Cada vez las instancias públicas están cambiando sus sistemas de medición de su rendimiento para encontrar fuentes de construcción de sus ventajas competitivas. Bonnefoy y Armijo (2005) plantean dos formas de indicadores de desempeño: indicadores según ámbito de medición e indicadores según sus dimensiones.

Los indicadores según el ámbito de medición son los siguientes:

- indicadores de insumos

- indicadores de proceso

- indicadores de producto

- indicadores de resultado final o impacto

El primero, está relacionado a cuánto recurso financiero se invirtió o cuánto recurso humano estuvo comprometido en la producción de bienes y servicios. Por ejemplo, número de familias implementadas con un fondo de capital semilla para negocios alternativos, o el porcentaje de presupuesto de capital semilla entrado a las familias, número de horas de trabajo del personal técnico especializado en el fortalecimiento de grupos solidarios. Así podemos ensayar varios ejemplos que den cuenta del uso de los recursos en el fortalecimiento de las familias. El segundo, muestra procesos, está relacionado a las formas o estrategias para hacer y lograr los objetivos, por ejemplo, número de días de demora para el abastecimiento de insumos veterinarios para el tratamiento de las enfermedades de los vacunos, o número de horas sin servicio de internet por mes. El tercero, se refiere a los indicadores que dan cuenta de los logros de la implementación de actividades o estrategias, por ejemplo, podemos indicar el porcentaje de niños vacunados contra la anemia, número de viviendas refaccionadas. Y el cuarto, da cuenta de los resultados esperados de un programa o proyecto implementado por la organización, por ejemplo, el porcentaje de líderes formados con capacidad de decisión, el porcentaje de incremento de ingresos familiares por la venta de textiles producidos con calidad; incluso, como indicador de efecto, tendríamos el número de mujeres líderes formadas con el proyecto, electas como autoridades provinciales. Sin embargo, de acuerdo al planteamiento de los mismos autores, esta forma de definir los 
indicadores tiene sus limitaciones, generalmente se restringe al tiempo de implementación o presencia de la organización en un determinado ámbito, no existe modo de medir fuera de ese tiempo, en el que se puede visibilizar efectos importantes como el desempeño de las mujeres líderes formadas como autoridades locales, o la mejoría de su calidad de vida a partir de los cambios ocurridos a largo plazo por las capacitaciones y las habilidades técnicas desarrolladas.

Indicadores según su dimensión de evaluación:

- eficacia

- eficiencia

- economía

- calidad

Estos cuatro indicadores están directamente relacionados al desempeño organizacional. El primero, concepto de eficacia, da cuenta del grado de cumplimiento de los objetivos planteados, es decir, en qué medida la organización o sus áreas específicas como un todo están cumpliendo con sus objetivos estratégicos, sin considerar los recursos asignados para ello. Y da cuenta solo del grado de cumplimiento de las metas u objetivos de la organización, desde su cobertura (número de actividades, el porcentaje de cobertura), focalización (análisis de la cobertura), capacidad de cubrir la demanda (en el tiempo y calidad) y el resultado final (mide la contribución, el efecto). A continuación, anotamos algunos ejemplos de indicadores de eficacia que hace al comportamiento de dos variables:

- número de ingresantes a la beca 18 del total de postulante;

- el porcentaje de las mujeres líderes certificadas del total de participantes en el programa;

- reducción del índice de mortalidad infantil por provincia.

El segundo indicador, concepto de eficiencia, hace referencia a dos elementos fundamentales, a la producción física de un bien o servicio con respecto a la menor cantidad de recurso utilizado, además, se identifica los factores que pueden estar generando un indicador que escape de la media de los estándares comparativos. En cuanto al análisis de la eficiencia económica, este se refiere a la adquisición y aprovechamiento de los insumos que deben ser adquiridos en el tiempo oportuno al costo aceptable. Los indicadores están planteados por tanto de la siguiente manera:

- costo del servicio técnico en agroecología / el número de usuarios que cuentan con áreas de cultivo de hortalizas;

- número de especialistas en salud/el número de la población usuaria del puesto de salud;

- número de viviendas evaluadas / número de evaluadores.

El tercer indicador, concepto de economía, refiere a la capacidad de la institución para movilizar los recursos financieros en pos del cumplimiento de los objetivos; hace hincapié en una administración responsable que la entidad debe realizar. Como ejemplos tenemos:

- cantidad de dinero gastado a través de compras en línea / total de compras realizadas;

- ingreso por prestación de servicios en calidad de consultores.

Finalmente, el cuarto indicador, concepto de calidad, hace referencia a la calidad de prestación de servicio, el que apunta a la capacidad de la organización para responder de manera rápida, oportuna y directa a las necesidades de los usuarios. Se considera como otros factores de calidad a la oportunidad, accesibilidad, precisión y continuidad en la entrega de los servicios, comodidad y cortesía en la atención.

Ejemplos de indicadores de calidad:

- porcentaje de reportes entregados en los plazos programados;

- número de pacientes atendidos por día;

- porcentaje de usuarios del servicio de telefonía Claro satisfechos;

- porcentaje de informes económicos anulados por error. 


\section{Importancia de la integración de los indicadores de desempeño}

Los indicadores, sean estos de insumo, productos, resultado final, por sí solos no muestran la verdadera dimensión que debe alcanzar la gestión de la entidad, es importante integrarlos con los indicadores de eficacia, eficiencia, económica y calidad a lo largo del proceso de la generación de bienes y servicios.

Algunos criterios desarrollados por Bonnefoy y Armijo (2005):

- Desarrollar los indicadores que cubran la totalidad de las dimensiones de desempeño: eficacia, eficiencia, económica y calidad.

- Definir indicadores, con resultados intermedios y finales, a ser desarrollados del conjunto de indicadores

- Generar indicadores cuyos efectos dependen de la acción transversal de varias instituciones públicas.

- Integrar los indicadores de proceso con los indicadores intermedios y finales.

En síntesis, estos indicadores deben cubrir diversas perspectivas de actuación de la organización, desde la perspectiva financiera del cliente, del proceso interno, humanas y de desarrollo.

\section{Construcción de los indicadores de desempeño}

Para el proceso de construcción de indicadores de desempeño no existe un patrón ni metodología establecida, lo único que asegura si está bien construido es la coherencia con el conjunto de procesos de mejora continua. Podríamos esbozar un conjunto de pasos:

- identificar o revisar productos y objetivos:

- identificar las dimensiones y ámbitos de desempeño;

- establecer responsabilidades organizacionales;

- establecer los referentes para la comparación;

- construir las fórmulas o algoritmos;

- recopilar la información necesaria;
- validar, aplicar criterios técnicos y requisitos;

- analizar los resultados obtenidos;

- comunicar e informar.

\section{Conclusiones}

Paraeldesempeñoactualdelasorganizaciones públicas, el establecer indicadores de desempeño se convierte, en términos de importancia, en un insumo sustancial para el proceso de cambio y la efectividad en su desarrollo. La entrega de los productos y servicios con calidad, implica una serie de cambios en los procesos administrativos, métodos, procesos de reflexión, innovación, como variables relevantes para alcanzar un óptimo desempeño.

El modelo tradicional burocrático de planificación quedó desfasado, ahora las organizaciones públicas deberían estar enfocadas en la gestión por resultados, generación de impactos relevantes, la misma que implica replantear sus procesos de planificación en el que se destaque las dimensiones de eficacia, eficiencia, económica, la calidad y empatía.

La búsqueda de la calidad en la gestión pública conduce a ser cuidadoso y preciso en la construcción de indicadores sin caer en las generalidades ni ambigüedades. Los indicadores de desempeño bien planteados garantizan la calidad de medición del desempeño organizacional.

\section{Referencias bibliográficas}

Allaire, Y. Y., \& Firsirotu, M. (1984). Theories of organizational culture. . In Organization Studies (3rd ed., Vol. 5, pp. 193-226). https:/l psycnet.apa.org/record/1987-29923-001

Bédard, R. (2004). Los fundamentos del Pensamiento y las Prácticas Administrativas. 2 - La trilogía Administrativa. AD-Minister, Universidad EAFIT, 2, 80-108.

Bonnefoy, J. y, \& Armijo, M. (2005). Indicadores de desempeño en el sector público. In Instituto Latinoamericano y del Caribe de Planificación Económica y Social - ILPES (Issue 1, pp. 45-106). $\quad$ http://repository.lasallista.edu.co/ dspace/bitstream/10567/720/1/Indicadores claves rendimiento CummisdelosAndes.pdf 
Carr, A. N. y, \& Hancock, P. (2006). Space and time in organizational change management. Journal of Organizational Change Management, 19(5), 545-557. https://doi. org $/ 10.1108 / 09534810610686058$

Fondas, N., \& Denison, D. R. (1991). Corporate Culture and Organizational Effectiveness: Is there a similar pattern around the world? In The Academy of Management Review (Vol. 16, Issue 1, pp. 205-227). https://doi. org $/ 10.2307 / 258613$

Gomez, C. F., \& Rodriguez, J. K. (2015). Teorías de la cultura organizacional. Revista Anthropologica, 5/6, 1-26. http://strathprints. strath.ac.uk/7361/

Martínez, M. E. (2010). Relaciones entre cultura y desempeño organizacional en una muestra de empresas Colombianas: reflexiones sobre la utilización del modelo de Denison. Cuadernos de Administracion, 23(40), 163190.

Ramírez, M. (2004). Desempeño Organizacional Retos $Y$ Enfoques Contemporáneos (1st ed., Vol. 1). Universidad de Occidente. https://books.google.com.mx/books?id= fpf7g2PhY6MC\&printsec=frontcover\&hl $=$ es $\&$ source $=$ gbs ge summary $\mathrm{r} \& \mathrm{cad}=0 \# \mathrm{~V}$ =onepage \& $q \& \mathrm{f}=$ false

Yamakawa, P., \& Ostos, J. (2011). Relación entre innovación organizacional y desempeño organizacional. Universidad \& Empresa, 13(21), 93-115. http://www.redalyc.org/ articulo.oa?id $=187222420005$ 